\title{
Softwarebezogene Wertschöpfung im Wertschöpfungsnetzwerk der Informations- und Kommunikationsindustrie
}

\author{
Eine empirische Analyse \\ Jochen Wulf, Rüdiger Zarnekow \\ Lehrstubl für Informations- und Kommunikationsmanagement, TU Berlin
}

\section{Einleitung}

Netzwerkphänomene wurden in der ökonomischen Forschung vielfach untersucht. Ein Großteil der Arbeiten fokussiert dabei auf Unternehmensnetzwerke. Diese bestehen aus zwischenbetrieblichen und häufig langfristigen Verbindungen von Unternehmen, die kooperativ Wert schöpfen (Norman und Ramirez 1993, Gulati et al. 2000, Jarillo 1988). Hierbei steht im Vordergrund, wie Rollen allokiert und die Zusammenarbeit zwischen Unternehmen gestaltet wird. Grundlegend für die Ausgestaltung von Unternehmensnetzwerken ist das Abhängigkeitsverhältnis von Wertschöpfungsaktivitäten, das in Wertschöpfungsnetzwerken abgebildet wird. In diesen werden die im Unternehmensnetzwerk zu verrichtenden Wertschöpfungsaktivitäten abgebildet und in Verbindung gesetzt (Porter und Millar 1985, Pil and Holweg 2006). Im Vordergrund steht hier also nicht die Rollenverteilung der Unternehmen sondern die zu verrichtenden Aktivitäten und deren Interrelationen.

Insbesondere der Analyse des Wertschöpfungsnetzwerks der Informationsund Kommunikationsindustrie kommt eine besondere Bedeutung zu, da hier aufgrund von technologischen und ökonomischen Einflüssen die Wertschöpfungsstruktur eine starke Dynamik besitzt (Basole 2009, Li und Whalley 2002, Turel and Yuan 2006). Viele wissenschaftliche Arbeiten beschreiben die sich verändernden Wertschöpfungsstrukturen anhand qualitativer Aussagen (Basole 2009. Fransman 2002, Li und Whalley 2002, Peppard and Rylander 2006, Zerdick et al. 2000, S. 130-135). Demgegenüber wurden quantitative Analysen zu Wertschöpfungsstrukturen in der Informations- und Kommunikationstechnik (IKT) Industrie, die in dieser Arbeit im Fokus stehen, in der wissenschaftlichen Literatur bisher spärlich publiziert. 
Durch Innovationen werden in der IKT Industrie neuartige Wertschöpfungskonstellationen geschaffen und zwingen Unternehmen, aus Wettbewerbsgründen strategische Neupositionierungen im Wertschöpfungsnetz vorzunehmen. Die Analyse und Identifikation von Aktivitätenassoziationen innerhalb von Wertschöpfungsnetzen ist für Unternehmen von strategischem Wert, weil sie den Handlungsraum von Unternehmen definieren, Wertschöpfungsprozesse transparent machen und die Identifikation von Unternehmensstrategien erleichtern.

So strebt beispielsweise British Telecom in einem aufgrund des Markteintrittes von Internet-Telefonie Anbietern veränderten IKT Wertschöpfungsnetz mit der Bereitstellung einer Entwicklungsplattform für Kommunikationsdienste im Internet und einer Abkehr vom Angebot vertikal integrierter Kommunikationsdienste eine Neupositionierung an (British Telecom 2009). Aus diesem Beispiel lässt sich eine wachsende Bedeutung der softwarebezogenen Wertschöpfung für die Telekommunikationsindustrie ableiten und mit Hilfe einer Wertschöpfungsanalyse genauer untersuchen. So können Rückschlüsse auf Strategien von Telekommunikations- und Softwareunternehmen gezogen werden (siehe Abschnitt 3.4).

In dieser Arbeit wird die Theorie der Wertschöpfungsnetzwerke und ihre Anwendung auf die IKT Industrie vorgestellt (Kapitel 2). Anschließend wird die Konsistenz des IKT Wertschöpfungsnetzes untersucht, um so den strategischen Entscheidungsraum von IKT Unternehmen auf klar definierte Wertschöpfungssegmente einzugrenzen. Des Weiteren wird in Kapitel 3 die Positionierung des Wertschöpfungssegmentes Software im IKT Wertschöpfungsnetz analysiert, um qualitative Aussagen über die Rolle der Softwarebranche für die IKT Industrie zu verifizieren. Im Fazit (Kapitel 4) werden Limitationen des methodischen Forschungsansatzes diskutiert und ein Ausblick auf zukünftige Forschungsaktivitäten gegeben.

\section{Theorie der Wertschöpfungsnetzwerke und ihre Anwendung auf die IKT Industrie}

\subsection{Das Wertschöpfungsnetzwerk als Handlungsfeld von Unternehmen}

Der Begriff der Wertschöpfungskette wurde von Porter geprägt. Er beschreibt die Gesamtheit der Primär- und Sekundäraktivitäten eines Unternehmens (Porter und Millar 1985). Primäraktivitäten tragen zu der Erstellung eines Produktes oder einer Dienstleistung unmittelbar bei, Sekundäraktivitäten stellen die Grundlagen zur Durchführung der Primäraktivitäten bereit. Unternehmensaktivitäten beeinflussen sich gegenseitig in ihren Kosten und ihrer Effektivität. Deshalb müssen Unternehmen Aktivitäten koordinieren und aufeinander abstimmen. Wertschöpfungsketten von Unternehmen derselben Industrie sind in einen größeren Aktivitätenfluss eingebettet, der von Porter und Millar (1985) als Value System bezeichnet wird. In diesem System werden Aktivitäten eines Unternehmens mit 
den Aktivitäten von Zulieferern und Kunden in Verbindung gesetzt. Die Art dieser Verbindungen und Abhängigkeiten determinieren dabei die Wettbewerbsposition eines Unternehmens, deren Ausgestaltung ist somit für Unternehmen von strategischem Wert.

Pil and Holweg (2006) beschreiben ein Wertschöpfungsnetz aus parallelen vertikalen Wertschöpfungsketten und horizontalen Wertschöpfungsstufen als potentielles zweidimensionales Handlungsfeld von Unternehmen. In diesem Handlungsfeld integrieren Unternehmen Wertschöpfungsaktivitäten, die in der Vertikalen, der Horizontalen oder der Diagonalen zueinander in Beziehung stehen. In vertikaler Richtung werden von einem Unternehmen auf der Nachfrager- oder Zuliefererseite Aktivitäten wahrgenommen, um auf die gesamte Wertschöpfungskette eine bessere Kontrolle ausüben zu können. In der Horizontalen werden Aktivitäten innerhalb einer Wertschöpfungsstufe ausgeführt, die auf verschieden Wertschöpfungsketten Einfluss nehmen. Hierdurch können Risiken diversifiziert und dabei bestehende Ressourcen effizienter eingesetzt sowie neuartige Produktund Dienstleistungsangebote erstellt werden. In der Diagonalen werden Aktivitäten in verschiedenen Wertschöpfungsketten und -stufen ausgeführt, um potentielle Versorgungsengpässe zu vermeiden oder positive Nachfrageeffekte auf das Kerngeschäft zu erzeugen. Tabelle 1 zeigt Beispiele für die verschiedenen Assoziationsarten von Wertschöpfungsaktivitäten.

Tabelle 1: Assoziationsarten von Wertschöpfungsaktivitäten (basierend auf Pil and Holweg 2006)

\begin{tabular}{|l|l|l|l|}
\hline $\begin{array}{l}\text { Assoziati- } \\
\text { onsart }\end{array}$ & $\begin{array}{l}\text { Unter- } \\
\text { nehmen }\end{array}$ & Wertschöpfungsaktivitäten & $\begin{array}{l}\text { Strategisches } \\
\text { Ziel }\end{array}$ \\
\hline Vertikal & Nokia & $\begin{array}{l}\text { Endgeräteherstellung, Mar- } \\
\text { kenbildung, Kundenbezie- } \\
\text { hungsmanagement }\end{array}$ & Kundenbindung \\
\hline Horizontal & $\begin{array}{l}\text { British } \\
\text { Telecom }\end{array}$ & $\begin{array}{l}\text { Mobile und Festnetzbasierte } \\
\text { Sprachdienste }\end{array}$ & $\begin{array}{l}\text { Wertschöpfungs- } \\
\text { integration }\end{array}$ \\
\hline Diagonal & Apple & $\begin{array}{l}\text { Endgeräteherstellung, Online } \\
\text { Musik Geschäft }\end{array}$ & $\begin{array}{l}\text { Erzeugung von } \\
\text { Kunden Lock-Ins }\end{array}$ \\
\hline
\end{tabular}

Wie aus der Arbeit von Pil and Holweg (2006) hervorgeht, sind Wertschöpfungsaktivitäten nicht isoliert zu betrachten sondern bilden ein komplexes und verwobenes Wertschöpfungsnetz. Den Positionierungsentscheidungen von Unternehmen innerhalb des Wertschöpfungsnetzes liegen wechselseitige Abhängigkeiten der integrierten Aktivitäten zugrunde, die ökonomisch begründet sind. Die Aufdeckung solcher Abhängigkeiten ist für Unternehmen von strategischem Wert, da sie Rückschlüsse auf Unternehmensstrategien und Wettbewerbssituationen zulässt und so für eine höhere Transparenz des Handlungsraumes von Unternehmen 
sorgen und eine Entscheidungsgrundlage zur Gestaltung von Unternehmensnetzwerken bietet.

\subsection{Das IKT Wertschöpfungsnetzwerk und die Rolle der Softwareindustrie}

Wie bereits im Konzept des Value Systems ersichtlich, lassen sich Wertschöpfungsaktivitäten gemäß ihres Abhängigkeitsgrades in branchenspezifische Wertschöpfungsnetzwerke abgrenzen und gruppieren. Eine solche Abgrenzung definiert den Aktionsbereich von Unternehmensnetzwerken und gibt einen Überblick über die strategisch relevanten Geschäftsfelder dazugehöriger Unternehmen.

Nach Porter und Millar (1985) lassen sich Aktivitäten von Unternehmen einer Branche zusammenfassen. Bezogen auf die Informations- und Kommunikationswirtschaft ist eine solche Gruppierung jedoch nicht eindeutig vorzunehmen: Aufgrund der in wissenschaftlicher Literatur viel zitierten Konvergenz der Branchen Telekommunikation, Software, Endgeräte und Medien (Li und Whalley 2002. Zerdick et al. 2000, S. 130-135) lassen sich hier klare Abgrenzungen nicht mehr vornehmen. Viele Forschungsarbeiten nehmen eine Neugruppierung der IKT Wertschöpfungsaktivitäten, Branchen und Firmen vor (Basole 2009. Fransman 2002, Li und Whalley 2002, Peppard and Rylander 2006, Zerdick et al. 2000, S. 130-135). Fransman (2002) präsentiert ein Ebenenmodell der IKT Aktivitäten und differenziert die Ebenen ,Equipment \& Software', ,Network, Connectivity', „Navigation \& Middleware' sowie ,Applications induding contents packaging'. Diese Arbeit beschreibt eine vertikale, kettenartige Anordnung von Wertschöpfungsaktivitäten aus den oben genannten Branchen. Direkte Abhängigkeiten bestehen vornehmlich innerhalb der Ebenen sowie zwischen Aktivitäten benachbarter Ebenen. Zerdick et al. (2000, S. 130-135) identifizieren einen fortgeschrittenen Prozess, der zur vollständigen Auflösung der Grenzen zwischen den Branchen Telekommunikation, Informationstechnologie (Software und Hardware) sowie Medien in einem Multimedia Markt führt. Im Gegensatz zu Fransman (2002) heben Zerdick et al. (2000) einen hohen Komplexitätsgrad des neu entstehenden Wertschöpfungssystems hervor: Im Multimedia Markt seien die ehemals vertikalen Wertschöpfungsaktivitäten stark miteinander verknüpft. Es wird eine generische Wertschöpfungskette vorgestellt, deren Einzelaktivitäten potentiell aus allen drei Sektoren bedient werden.

Die zitierten Arbeiten zur Konvergenz fokussieren nicht speziell auf dem Software Sektor, jedoch werden in ihnen softwarebezogene Wertschöpfungsaktivitäten als Bestandteile der IKT Dienstleistungsproduktion angesehen. Die Integration und Koordination der einzelnen Wertschöpfungsaktivitäten wird als eigenständige Aktivität aufgefasst und als Dienstintegration und Dienstbereitstellung bezeichnet. In ihrer Analyse des Software-Wertschöpfungsnetzwerkes hebt Helander (2004, S. 23 und S. 67) die wichtige Rolle der mit Softwareproduktion und Anwendungsbetrieb verbundenen Aktivitäten für andere Industrien, vor allen Dingen in den Bereichen Industrielle Automatisierung, Telekommunikation und 
Elektronik hervor. Die Entwicklung und Bereitstellung softwarebasierter Dienste und Anwendungen in diesen Bereichen werde als Katalysator des Kerngeschäftes angesehen. Dies drückte sich unter Anderem darin aus, dass viele Unternehmen, deren Kerngeschäft nicht im Softwarebereich einzuordnen ist, zusätzlich Software entwickeln und vertreiben. Für Hardwareequipmenthersteller ist Software ein Komplementärprodukt, da nur die Kombination von Wertschöpfungsaktivitäten beider Segmente Nutzen stiften kann (Cottrell und Koput 1998). In der Telekommunikationsbranche spielt Software aufgrund von Konvergenzeffekten eine zunehmend wichtige Rolle. So stellt Messerschmitt (1996) fest, dass Telekommunikationsanwendungen sich von ,Networked Computing' Anwendungen nicht mehr unterscheiden und deshalb Anwendungen vermehrt softwarebasiert auf programmierbaren Endgeräten umgesetzt werden. Internet Telefonie ist hierfür ein gutes Beispiel. In der Medienbranche werden softwarebasierte Systeme unter Anderem zur Personalisierung von Inhalten, zur Erschließung elektronischer Vertriebskanäle und zur Ausgestaltung differenzierter Preisstrategien benötigt (Schumann und Hess 2006, S. 49-79).

\section{Empirische Untersuchung}

Die im vorigen Abschnitt vorgestellten Aussagen zur Entwicklung des IKT Wertschöpfungsnetzwerkes und der Position der dem Softwaresektor zuzuordnenden Aktivitäten in diesem Netzwerk sind überwiegend qualitativer Natur. In diesem Kapitel wird eine empirische Analyse des IKT Wertschöpfungsnetzwerkes durchgeführt. Im Fokus steht insbesondere die Wertschöpfung der Softwarebranche.

\subsection{Forschungsthesen}

Aus den vorhandenen wissenschaftlichen Arbeiten zum IKT Wertschöpfungsnetzwerk lassen sich Hypothesen ableiten, die in der quantitativen Auswertung untersucht werden sollen. Die Hypothesen H1 und H2 sind konstitutiv für die Existenz eines IKT Wertschöpfungsnetzwerks:

- H1: Zwischen den Wertschöpfungsaktivitäten der Segmente Hardware Komponenten, Hardware Equipment, Software, Telekommunikation und Medien bestehen starke Abbängigkeiten

- H2: Als IKT Wertschöpfungsnetzperk lassen sich diese Aktivitäten klar von anderen Wertschöpfungsaktivitäten abgrenzen.

Die dritte Hypothese H3 beschreibt die wichtige Rolle des Softwaresektors im IKT Wertschöpfungsnetzwerk zur Bereitstellung von IKT Endprodukten und Dienstleistungen. 
- H3: Die Wertschöpfungsaktivitäten des Segmentes Software stehen in starker Verbindung zu den Segmenten Hardware, Medien sowie Telekommunikation, deren Wertschöpfung sich in einem signifikanten Abhängigkeitsverbältnis zum Softwaresegment befindet.

Ziel der nachfolgenden Ausführungen ist die Untersuchung dieser Hypothesen: im anschließenden Abschnitt werden Datenbasis und Untersuchungsmethode vorgestellt. Die Hypothesen $\mathrm{H} 1$ und $\mathrm{H} 2$ werden in Abschnitt 3.3 untersucht, $\mathrm{H} 3$ in Abschnitt 3.4.

\subsection{Datenbasis und Untersuchungsmethode}

Als Datenbasis für die folgende Analyse dient ein Datensatz der Datenbank Thomson ONE Banker, der für 34142 börsennotierte Unternehmen bis zu 8 Standard Industrial Classification (SIC) Codes aufführt, die absteigend geordnet nach ihrem Umsatzanteil Wertschöpfungsaktivitäten von Unternehmen beschreiben. Wertschöpfungsaktivitäten wurden dabei in Segmente eingeteilt. Tabelle 2 beschreibt die Klassifizierung der dem IKT Wertschöpfungsnetzwerk zugehörigen Wertschöpfungssegmente.

Um Aussagen über die wechselseitigen Beziehungen von Wertschöpfungssegmenten treffen zu können, wurde folgendes Assoziationsmaß definiert:

$$
z(a, b)=\mid A \text { AND } B|/| A O R B \mid
$$

Hierbei ist A (B) die Menge aller Firmen, die im Wertschöpfungssegment a (b) aktiv sind. Die Funktion $z(\mathrm{a}, \mathrm{b})$ setzt die Anzahl der Firmen, die gleichzeitig in a und $\mathrm{b}$ aktiv sind, in Beziehung zu der Anzahl aller Firmen, die in a oder b aktiv sind. Diesem Assoziationsmaß liegt das im Abschnitt 2.1 formulierte Prinzip zugrunde, dass die Motivation zur Integration von Wertschöpfungsaktivitäten durch die Assoziation der Wertschöpfungsaktivitäten begründet ist.

Basierend auf dem Assoziationsgrad der einzelnen Wertschöpfungssegmente wurde im ersten Schritt eine Clusteranalyse (Everitt 1993) durchgeführt. Ziel dieser Analyse war die Gruppierung von Wertschöpfungssegmenten, die ein ähnliches Assoziationsprofil aufweisen: Wertschöpfungssegmente, zwischen denen ein starker Zusammenhang besteht und die sich gleichzeitig von außenstehenden Segmenten gut abgrenzen lassen, wurden dabei in Gruppen zusammengefasst. Hierzu wurde eine hierarchische Clusteranalyse mit einer Cluster-Methode durchgeführt, die das arithmetische Mittel der Distanzen aller Objekte zweier Cluster als Maß für die Distanz zwischen diesen Clustern verwendet (,Linkage zwischen den Gruppen') und als Distanzmaß die quadratische euklidische Distanz benutzt. Da der flache Verlauf der durchschnittlichen Distanz zwischen den Clustern in Abhängigkeit von der Clusteranzahl die Bestimmung einer optimalen Clusteranzahl gemäß des Ellbow-Kriteriums (Everitt 1993, S. 100) nicht zulässt, wurden aus Gründen 
der Übersichtlichkeit neun Cluster erzeugt. In Abschnitt 3.3 werden die Ergebnisse der Clusteranalyse vorgestellt.

Tabelle 2: IKT Wertschöpfungssegmente

\begin{tabular}{|c|c|c|c|}
\hline $\begin{array}{l}\text { Seg- } \\
\text { ment }\end{array}$ & Beschreibung & Beispiele & $\begin{array}{l}\text { SIC Kodierung (Aus- } \\
\text { wahl) }\end{array}$ \\
\hline $\begin{array}{l}\text { Hard- } \\
\text { ware } \\
\text { Kom- } \\
\text { ponen- } \\
\text { ten }\end{array}$ & $\begin{array}{l}\text { Produktion von Mate- } \\
\text { rial und Komponen- } \\
\text { ten, die zur Herstel- } \\
\text { lung von Hardware- } \\
\text { equipment benötigt } \\
\text { wird }\end{array}$ & $\begin{array}{l}\text {-Halbleiter } \\
\text {-Drähte und Leitungen }\end{array}$ & $\begin{array}{l}3671,3672,3674,3675, \\
3676,3677,3678,3679, \\
3691,3692,3694,3695, \\
3699\end{array}$ \\
\hline $\begin{array}{l}\text { Hard- } \\
\text { ware } \\
\text { Equip- } \\
\text { ment }\end{array}$ & $\begin{array}{l}\text { Produktion von } \\
\text { Kommunikationsend- } \\
\text { geräten und Netzwerk- } \\
\text { equipment }\end{array}$ & $\begin{array}{l}\text {-Computer } \\
\text {-Mobiltelefonie } \\
\text {-Router }\end{array}$ & $\begin{array}{l}3571,3572,3575,3577, \\
3578,3579,3651,3652, \\
3661,3663,3669,5045, \\
5063,5064,5065, \ldots\end{array}$ \\
\hline $\begin{array}{l}\text { Soft- } \\
\text { ware }\end{array}$ & $\begin{array}{l}\text { Entwicklung von } \\
\text { Software und Internet- } \\
\text { anwendungen sowie } \\
\text { damit verbundene } \\
\text { Beratungs- und Aus- } \\
\text { bildungsleistungen }\end{array}$ & $\begin{array}{l}\text {-Softwareentwicklung } \\
\text {-Informationsdienste }\end{array}$ & $\begin{array}{l}\text { 7370, 7371, 7372, 7373, } \\
7374,7375,7379,7382\end{array}$ \\
\hline $\begin{array}{l}\text { Tele- } \\
\text { kom- } \\
\text { muni- } \\
\text { kation }\end{array}$ & $\begin{array}{l}\text { Bereitstellung von } \\
\text { Kommunikations- } \\
\text { diensten sowie Netz- } \\
\text { management und - } \\
\text { betrieb }\end{array}$ & $\begin{array}{l}\text {-PSTN und GSM Tele- } \\
\text { fonie } \\
\text {-DSL Internet Zugang }\end{array}$ & $\begin{array}{l}\text { 4810, 4812, 4813, 4822, } \\
4832,4833,4841,4899\end{array}$ \\
\hline Medien & $\begin{array}{l}\text { Produktion und Mana- } \\
\text { gement von Text, } \\
\text { grafischen und multi- } \\
\text { medialen Inhalten }\end{array}$ & $\begin{array}{l}\text {-Publikation von Zei- } \\
\text { tungen } \\
\text {-Werbedienste } \\
\text {-Filmproduktion }\end{array}$ & $\begin{array}{l}2711,2721,2731,2732, \\
2741,2752,2754,2759, \\
2761,2771,2782,2789, \\
2791,2796,7311,7312, \\
7313,7319,7331, \ldots\end{array}$ \\
\hline
\end{tabular}

Anschließend wurde mit Hilfe von Kontingenzanalysen (Wickens 1989, S. 17-50) die Positionierung des Wertschöpfungssegmentes Software im IKT Wertschöpfungsnetz genauer untersucht. Im Fokus dieser Untersuchung stand die Fragestellung, ob Firmen, die im Segment Software aktiv sind, sich im Bezug auf ihr Engagement in weiteren Segmenten des IKT Wertschöpfungsnetzwerks signifikant von anderen IKT-Firmen unterscheiden. Um signifikante Assoziationen zwischen der Softwareaktivität und Aktivitäten in weiteren Segmenten zu ermitteln, wurden $\mathrm{X}^{2}$ Tests nach Pearson durchgeführt sowie Kontingenzkoeffizienten berechnet. Im Abschnitt 3.4 werden die Ergebnisse der Kontingenzanalyse vorgestellt. 


\subsection{Identifikation des IKT Wertschöpfungsnetzwerkes}

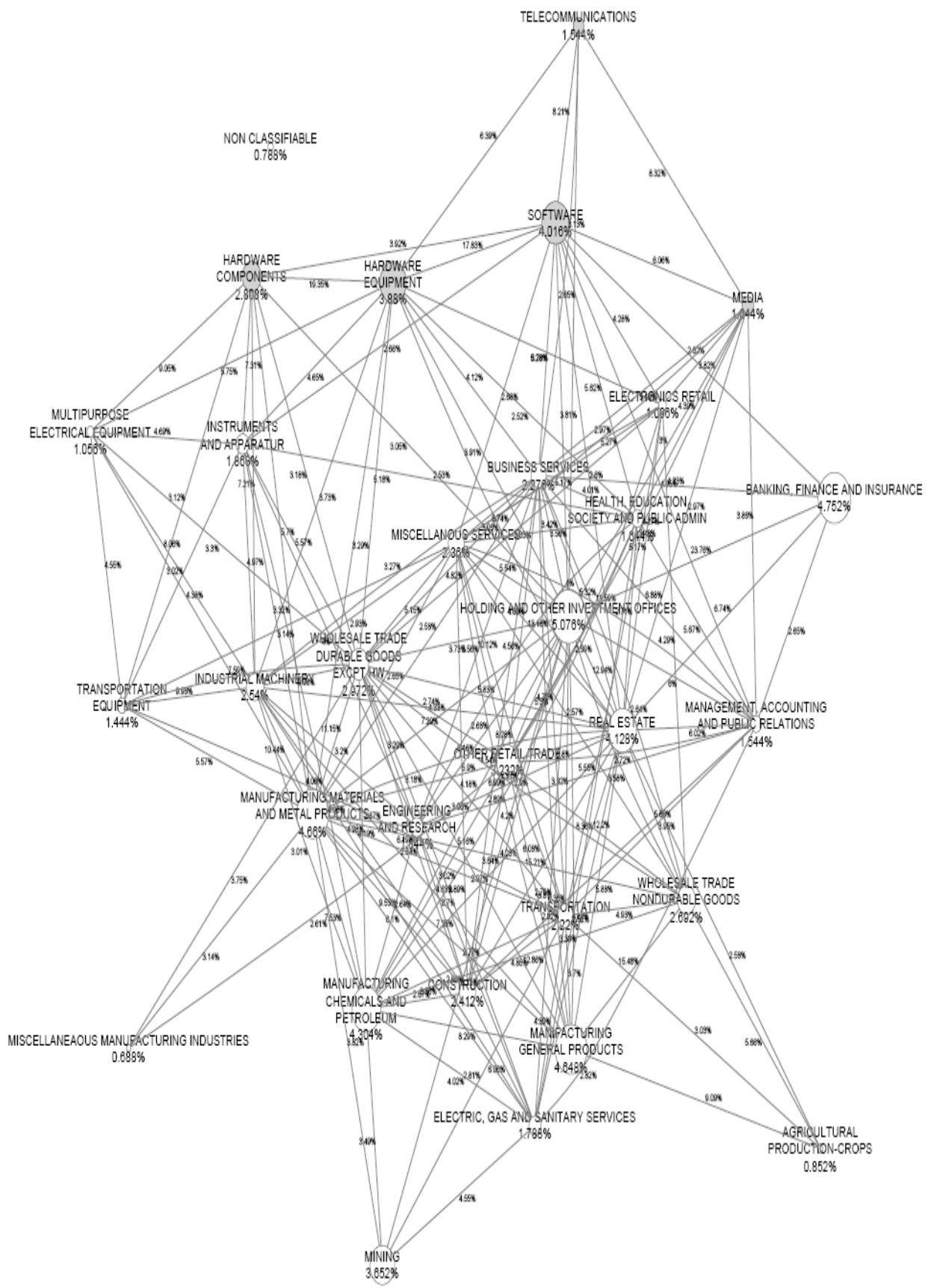

Abbildung 1: Visualisierung des gesamten Wertschöpfungsnetzwerks 
Die Assoziationen zwischen den Wertschöpfungssegmenten wurde mit Hilfe der Visualisierungssoftware Graphviz (Ellson et al. 2004) grafisch dargestellt. Diese benutzt Verfahren der multidimensionalen Skalierung, um Knoten gemäß der Länge der kürzesten Pfade anzuordnen. So werden Cluster als Netzwerk benachbarter Knoten sichtbar.

Tabelle 3: Gruppierung der Wertschöpfungssegmente

\begin{tabular}{|c|c|}
\hline Cluster & Wertschöpfungssegmente \\
\hline 1 & $\begin{array}{l}\text { HARDWARE COMPONENTS, HARDWARE EQUIPMENT, } \\
\text { SOFTWARE, TELECOMMUNICATIONS, MEDIA }\end{array}$ \\
\hline 2 & $\begin{array}{l}\text { ELECTRONICS RETAIL, CONSTRUCTION, TRANSPORTA- } \\
\text { TION, OTHER RETAIL TRADE, REAL ESTATE, MISCEL- } \\
\text { LANOUS SERVICES }\end{array}$ \\
\hline 3 & $\begin{array}{l}\text { AGRICULTURAL PRODUCTION-CROPS, MANIFACTURING } \\
\text { GENERAL PRODUCTS , MANUFACTURING CHEMICALS } \\
\text { AND PETROLEUM, WHOLESALE TRADE NONDURABLE } \\
\text { GOODS }\end{array}$ \\
\hline 4 & MINING, ELECTRIC, GAS AND SANITARY SERVICES \\
\hline 5 & $\begin{array}{l}\text { MANUFACTURING MATERIALS AND METAL PRODUCTS, } \\
\text { INDUSTRIAL MACHINERY, MULTIPURPOSE ELECTRICAL } \\
\text { EQUIPMENT, TRANSPORTATION EQUIPMENT, INSTRU- } \\
\text { MENTS AND APPARATUR, WHOLESALE TRADE DURABLE } \\
\text { GOODS EXCPT HW }\end{array}$ \\
\hline 6 & MISCELLANEAOUS MANUFACTURING INDUSTRIES, \\
\hline 7 & $\begin{array}{l}\text { BANKING, FINANCE AND INSURANCE, HOLDING AND } \\
\text { OTHER INVESTMENT OFFICES }\end{array}$ \\
\hline 8 & $\begin{array}{l}\text { BUSINESS SERVICES, ENGINEERING AND RESEARCH, } \\
\text { MANAGEMENT, ACCOUNTING AND PUBLIC RELATIONS, } \\
\text { HEALTH, EDUCATION, SOCIETY AND PUBLIC ADMIN }\end{array}$ \\
\hline 9 & NON CLASSIFIABLE \\
\hline
\end{tabular}

Die Größe der Knoten repräsentiert die Anzahl der Unternehmen, die diesem Wertschöpfungssegment zugeordnet sind. Die Kantenlänge beschreibt den Grad der Assoziation zwischen zwei Wertschöpfungssegmenten. Um die Darstellung übersichtlich zu gestalten, wurden nur Assoziationen berücksichtigt, die einen Schwellwert von 2,5\% nicht unterschreiten. Wie in Abbildung 1 veranschaulicht, werden die fünf IKT Aktivitätssegment (in der oberen Bildhälfte gekennzeichnet durch eine graue Füllung) in unmittelbarer Nachbarschaft zueinander angeordnet 
und durch die Positionierung am oberen Rand des gesamten Netzwerks von den anderen Aktivitäten abgegrenzt.

Das Ergebnis der Clusteranalyse bestätigt die beiden konstitutiven Eigenschaften des IKT Wertschöpfnetzes (Thesen H1 und H2). Tabelle 3 enthält die resultierende Clustereinteilung der Wertschöpfungssegmente. Das Cluster Nummer 1 repräsentiert dabei das IKT Wertschöpfungsnetz. Somit ist es gelungen, die qualitativen Aussagen früherer Arbeiten bezüglich der Existenz des IKT Wertschöpfungsnetzes mit Hilfe eines quantitativen Verfahrens zu bestätigen. Diese Ergebnisse rechtfertigen auch die weitgehende Beschränkung der Analyse der Position des Wertschöpfungssegmentes Software auf die Assoziation zu anderen IKT Wertschöpfungssegmenten im anschließenden Abschnitt.

\subsection{Wertschöpfung der Softwareindustrie im IKT Wertschöpfungsnetzwerk}

Um Signifikanzen in Assoziationen des Wertschöpfungssegmentes Software mit anderen IKT Wertschöpfungssegmenten festzustellen, wurden die Komplementäraktivitäten (d.h. die zusätzlichen Aktivitäten) von Softwarefirmen mit Hilfe von Kontingenzanalysen den Komplementäraktivitäten von anderen IKT-Firmen gegenübergestellt. Tabelle 4 fasst die Analyseergebnisse zusammen: in Spalte zwei wird deutlich, dass Firmen, die sich im Softwarebereich engagieren, seltener in der Produktion von Hardware Komponenten tätig sind (6,4\%) als andere IKT Firmen, die sich in den Segmenten Hardwareequipment, Telekommunikation oder Medien engagieren (21,2\%). Durch den $\mathrm{X}^{2}$-Test kann die Hypothese des Nicht-Bestehens dieses Assoziationsunterschiedes bei einem Signifikanzniveau von 99\% abgelehnt werden.

Tabelle 4: Kontingenzanalysen zur Positionierung des Wertschöpfungssegmentes Software im IKT Wertschöpfungsnetz

\begin{tabular}{|l|l|l|l|l|}
\hline & $\begin{array}{l}\text { Hardware } \\
\text { Components }\end{array}$ & $\begin{array}{l}\text { Hardware } \\
\text { Equipment }\end{array}$ & $\begin{array}{l}\text { Telecom- } \\
\text { munications }\end{array}$ & Media \\
\hline $\begin{array}{l}\text { Anzahl von Softwarefir- } \\
\text { men mit Komplementär- } \\
\text { aktivität in }\end{array}$ & $220(6,4 \%)$ & $\begin{array}{l}1020 \\
(29,7 \%)\end{array}$ & $360(10,5 \%)$ & $\begin{array}{l}276 \\
(8,0 \%)\end{array}$ \\
\hline $\begin{array}{l}\text { Anzahl von Nicht- } \\
\text { Software-IKT-Firmen mit } \\
\text { Komplementäraktivität in }\end{array}$ & $844(21,2 \%)$ & $\begin{array}{l}1006 \\
(24,9 \%)\end{array}$ & $346(7,4 \%)$ & $\begin{array}{l}215 \\
(4,9 \%)\end{array}$ \\
\hline $\mathrm{X}^{2}$ (Pearson) & $326,986^{*}$ & $21,607^{*}$ & $24,807^{*}$ & $33,003^{*}$ \\
\hline $\mathrm{C}$ & 0,206 & 0,054 & 0,055 & 0,065 \\
\hline
\end{tabular}

$*: \mathbf{X}^{2}>9.210$ 
Die geringe Assoziation zwischen Software- und Hardwarekomponentensegment wird insbesondere durch einen Vergleich von Software- und Hardwareequipmentsegment plausibel: in der Produktion von Endgeräten werden Hardwarekomponenten direkt benötigt. Deshalb kann eine gleichzeitige Kontrollausübung über beide Aktivitäten von strategischem Wert sein. Hingegen sind Synergieeffekte zwischen Komponentenherstellung und softwarespezifischen Aktivitäten schwerer auszumachen. Jedoch weist der Kontingenzkoeffizient $\mathrm{C}$ auf keinen starken Assoziationsunterschied hin.

Mit den Segmenten Hardwareequipment, Telekommunikation und Medien (Spalten 3-5) weist das Softwaresegment einen stärkeren Assoziationsgrad auf als die anderen IKT Segmente. Zwar lassen sich mit Hilfe des Kontingenzkoeffizienten keine starken Assoziationsunterschiede nachweisen, jedoch lässt sich mit Hilfe des $\mathrm{X}^{2}$-Tests auch keine Gleichartigkeit der Assoziationsgrade feststellen. Auch wenn sich die Validität der Hypothese $\mathrm{H} 3$ also nicht durch Abgrenzung des Softwaresegmentes von anderen IKT-Wertschöpfungssegmenten in einer Kontingenzanalyse statistisch nachweisen lässt, bestätigen die beobachteten Assoziationsmaße (Zeile 2 in Tabelle 4), dass sich Softwarefirmen im Vergleich zu IKT Firmen, die keine Softwareaktivitäten ausführen, überdurchschnittlich häufig auch in den Segmenten Hardwareequipment, Telekommunikation und Medien engagieren.

Um Erkenntnisse über die Richtung der Assoziation vom Softwaresegment mit anderen Wertschöpfungssegmenten zu erhalten, wurden Firmen im Gegensatz zu den vorherigen Analysen den einzelnen Segmenten ausschließlich auf Basis ihrer primären SIC Kodierung (Primäraktivität) zugeordnet. Hierbei gehen die Autoren von der Prämisse aus, dass eine Sekundäraktivität aufgrund ihres betriebswirtschaftlich fundierten Einflusses auf die Primäraktivität von Firmen ausgeführt wird. Auch wenn diese Prämisse keinen allgemeinen Geltungsanspruch besitzt, so wird sie von den Ausführungen in Abschnitt 2.1 gestützt. Abbildung 2 zeigt die Ergebnisse dieser Analyse. Die Breite eines Pfeiles $(a \rightarrow b)$ stellt dabei den Anteil der Firmen eines Primärwertschöpfungssegments (a) dar, die mit Nebenaktivitäten in einem anderen Segment (b) unternehmerisch aktiv ist. Es wurden hierbei nur Segmente miteinbezogen, in denen mindesten 5\% der Firmen Nebenaktivitäten im Bereich Software aufweisen, oder in denen Softwarefirmen zu mindestens $5 \%$ mit Nebentätigkeiten aktiv sind. Neben dem starken gegenseitigen Abhängigkeitsverhältnis mit dem ,Hardware Equipment' Sektor fallen hierbei vor allem die starken einseitigen Abhängigkeiten der Segmente ,Telecommunications', ,Management, Accounting and Public Relations' sowie ,Media' vom Softwaresegment auf. Diese Beobachtung bestätigt die Hypothese H3 in Bezug auf die gerichtete Abhängigkeit der Segmente Hardware Equipment, Telekommunikation und Medien vom Softwaresegment.

Während sich beispielsweise Telekommunikationsunternehmen stark im Softwaresegment engagieren, ist dies umgekehrt nicht der Fall. Hieraus lässt sich ableiten, dass softwarebezogene Komplementäraktivitäten für Telekommunikationsunternehmen strategischen Wert besitzen, telekommunikationsbezogene Komple- 
mentäraktivitäten für Softwareunternehmen jedoch nicht. Wie vielfältige Beispiele, etwa das Engagement von Netzbetreibern bei der Entwicklung des Betriebssystems Android (Open Handset Alliance 2009) oder der Einsatz des IPTV Systems Mediaroom der Firma Microsoft beim IPTV-Angebot T-Home Entertain der Deutschen Telekom (Microsoft 2008), zeigen, beruht die Erstellung von Dienstleistungen im Bereich Telekommunikation vielfach auf dem Beitrag softwarebezogener Wertschöpfungsaktivitäten.

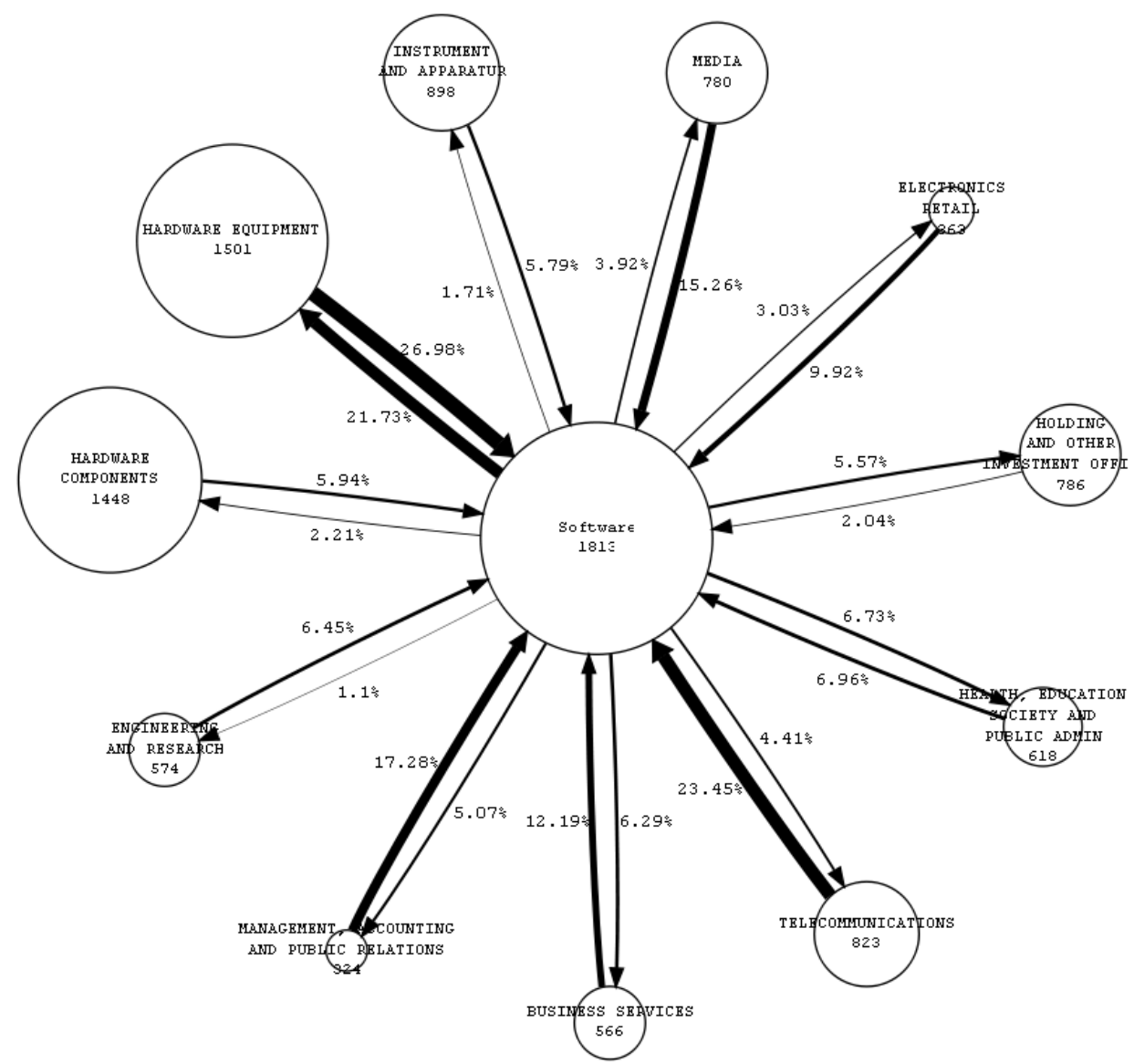

Abbildung 2: Abhängigkeitsverhältnis des Wertschöpfungssegmentes Software zu anderen Wertschöpfungssegmenten

Insbesondere aufgrund der Entwicklung des Internets lassen sich für Softwareunternehmen neue Geschäftsfelder erschließen. So können beispielsweise Medienund Telekommunikationsdienste softwaregestützt über das Internet bereitgestellt werden. Wie aus Abbildung 2 hervorgeht, werden Kompetenzen in der Softwareentwicklung und im Softwarebetrieb in vielfältigen Branchen benötigt. Softwareun- 
ternehmen können über die enge Bindung mit Hardwareequipmentherstellern hinaus unabhängig agieren und ihre Wertschöpfung flexibel in benachbarten Branchen einbringen. Gleichzeitig werden Unternehmen benachbarter Branchen im Softwaresegment aktiv und verändern die marktspezifische Wettbewerbssituation. So bietet zum Beispiel die Detecon International GmbH, die ursprünglich als Beteiligung der Deutschen Bundespost zur Erbringung von Beratungsdienstleistungen im Bereich der Fernmeldetechnik gegründet wurde, heute auch IT Beratungsleistungen an (Detecon 2009).

\section{$4 \quad$ Fazit}

In dieser Arbeit wurde basierend auf Daten über Wertschöpfungsaktivitäten von Unternehmen das IKT Wertschöpfungsnetzwerk analysiert. Dabei konnten die vielfach nur qualitativ vorgenommenen Aussagen über die Existenz eines IKT Wertschöpfungsnetzes bestehend aus Wertschöpfungsaktivitäten der Segmente Hardwarekomponenten, Hardwareequipment, Software, Telekommunikation und Medien empirisch bestätigt werden. Des Weiteren konnte gezeigt werden, dass das Wertschöpfungssegment Software eine zentrale Position im IKT Wertschöpfungsnetz einnimmt: insbesondere für Firmen, die Wertschöpfung in den Segmenten Hardwareequipment, Medien und Telekommunikation durchführen, stellen Softwareaktivitäten wichtige Komplementäraktivitäten dar.

Basierend auf dem in dieser Arbeit verwendeten Datensatz können Assoziationen zwischen Aktivitäten nur aufgrund von innerbetrieblich integrierter Wertschöpfungen identifiziert werden. Zwischenbetriebliche Kooperationen weisen ebenfalls auf Aktivitätenassoziationen hin. Des Weiteren wurden bei dieser Analyse lediglich umsatzerzeugende Wertschöpfungsaktivitäten berücksichtigt, nicht jedoch innerbetriebliche Aktivitäten, die keinen außerbetrieblichen Wert darstellen. Auch wenn diese Limitationen die Aussagekraft der erzielten Ergebnisse schmälern, so stellen sie die Plausibilität der Ergebnisse dennoch nicht in Frage, da bei der Definition des Assoziationsmaßes auf die betriebswirtschaftlichen Argumente zur innerbetrieblichen Integration von umsatzerzeugenden Wertschöpfungsaktivitäten fokussiert wurde.

Im Rahmen der in dieser Arbeit vorgestellten Analyse wurden Wertschöpfungsaktivitäten im IKT Sektor in grob-granulöse Segmente eingeteilt. Diese Einteilung war für die Untersuchung der diskutierten Hypothesen zweckmäßig, lässt allerdings tiefer gehende Aussagen bezüglich der Assoziationen von Wertschöpfungsaktivitäten und der Zusammensetzung des IKT Wertschöpfungsnetzwerkes nicht zu. Deshalb begründet diese Arbeit weitere, auf das IKT Wertschöpfungsnetzwerk beschränkte Analysen basierend auf fein-granulösen Wertschöpfungssegmenten. 


\section{Literatur}

Basole RC (2009) Visualization of Interfirm Relations in a Converging Mobile Ecosystem. Journal of Information Technology 24(2):144-159.

British Telecom (2009) Ribbit Developer Platform. http://developer.ribbit.com. Abruf am 2009-09-11.

Cottrell T, Koput K (1998) Software variety and hardware value: a case study of complementary network externalities in the microcomputer software industry. Journal of Engineering and Technology Management 15(4):309-338.

Detecon (2009) Detecon Historie. http://www.detecon.com/de/ueber_detecon/historie.html. Abruf am 200909-08.

Ellson J, Gansner ER, Koutsofios E (2004) Graphviz and dynagraph static and dynamic graph drawing tools. Technical report, AT\&T Labs - Research, Florham Park NJ 07932, USA.

Everitt BS (1993) Cluster Analysis. 3. Aufl. Edward Arnold, London.

Fransman M (2002) Mapping the evolving telecoms industry: the uses and shortcomings of the layer model. Telecommunications Policy 26(9-10):473483.

Gulati R, Nohria N, Zaheer A (2000) Strategic networks. Strategic Management Journal 21(3):203-215.

Helander N (2004) Value-creating networks: an analysis of the software component business. PhD Thesis, Acta Universitatis Ouluensis.

Jarillo JC (1988) On strategic networks. Strategic Management Journal 9(1):31-41.

Li F, Whalley J (2002) Deconstruction of the telecommunications industry: from value chains to value networks. Telecommunications Policy 260-10):451-472.

Messerschmitt DG (1996) The convergence of telecommunications and computing: what are the implications today?. In Proceedings of the IEEE 84(8):1167-1186.

Microsoft (2008) CeBIT 2008: Microsoft und Deutsche Telekom präsentieren IPTV-Angebot. http://www.microsoft.com/germany/presseservice/detail.mspx?id=532130. Abruf am 2009-09-21.

Norman R, Ramirez R (1993) From Value Chain to Value Constellation: Designing Interactive Strategy. Long Range Planning 26(6):151-151. 
Open Handset Alliance (2009) Android | Official Website.

http://www.android.com. Abruf am 2009-09-18.

Peppard J, Rylander A (2006) From Value Chain to Value Network: Insights for Mobile Operators. European Management Journal 24(2-3):128-141.

Pil FK, Holweg M (2006) Evolving from Value Chain to Value Grid. MIT Sloan Management Review 47(4):72-79.

Porter ME, Millar V (1985) How information gives you competitive advantage. Harvard Business Review 63(4):149-160.

Schumann M, Hess T (2006) Grundfragen der Medienwirtschaft: eine betriebswirtschaftliche Einführung. 3. Aufl. Springer, Berlin.

Turel O, Yuan Y (2006) Investigating the dynamics of the m-commerce value system: a comparative viewpoint. International Journal of Mobile Communications 4(5):532-557.

Wickens TD (1989) Multiway contingency tables analysis for the social sciences. Lawrence Erlbaum, Hillsdale.

Zerdick A, Picot A, Schrape K (2000) E-Conomics: Strategies for the Digital Marketplace. Springer, Berlin. 